\title{
Surgical outcomes of Majewski osteodysplastic primordial dwarfism Type II with intracranial vascular anomalies
}

\author{
Mario Teo, MD, FRCS(SN), ${ }^{1}$ Jeremiah N. Johnson, MD, ${ }^{1}$ Teresa E. Bell-Stephens, RN, ${ }^{1}$ \\ Michael P. Marks, MD, ${ }^{1,2}$ Huy M. Do, MD, ${ }^{1,2}$ Robert L. Dodd, MD, PhD, ${ }^{1,2}$ Michael B. Bober, MD, PhD, ${ }^{3}$ \\ and Gary K. Steinberg, MD, PhD'
}

Departments of ${ }^{1}$ Neurosurgery and ${ }^{2}$ Radiology, Stanford University Medical Center, Palo Alto, California; and ${ }^{3}$ Division of Genetics, Department of Pediatrics, Nemours/Alfred I. duPont Hospital for Children, Wilmington, Delaware

OBJECTIVE Majewski osteodysplastic primordial dwarfism Type II (MOPD II) is a rare genetic disorder. Features of it include extremely small stature, severe microcephaly, and normal or near-normal intelligence. Previous studies have found that more than $50 \%$ of patients with MOPD II have intracranial vascular anomalies, but few successful surgical revascularization or aneurysm-clipping cases have been reported because of the diminutive arteries and narrow surgical corridors in these patients. Here, the authors report on a large series of patients with MOPD II who underwent surgery for an intracranial vascular anomaly.

METHODS In conjunction with an approved prospective registry of patients with MOPD II, a prospectively collected institutional surgical database of children with MOPD II and intracranial vascular anomalies who underwent surgery was analyzed retrospectively to establish long-term outcomes.

RESULTS Ten patients with MOPD II underwent surgery between 2005 and 2012; 5 patients had moyamoya disease (MMD), 2 had intracranial aneurysms, and 3 had both MMD and aneurysms. Patients presented with transient ischemic attack $(T I A)(n=2)$, ischemic stroke $(n=2)$, intraparenchymal hemorrhage from MMD $(n=1)$, and aneurysmal subarachnoid hemorrhage $(n=1)$, and 4 were diagnosed on screening. The mean age of the 8 patients with MMD, all of whom underwent extracranial-intracranial revascularization (14 indirect, 1 direct) was 9 years (range 1-17 years). The mean age of the 5 patients with aneurysms was 15.5 years (range $9-18$ years). Two patients experienced postoperative complications (1 transient weakness after clipping, 1 femoral thrombosis that required surgical repair). During a mean follow-up of 5.9 years (range 3-10 years), 3 patients died (1 of subarachnoid hemorrhage, 1 of myocardial infarct, and 1 of respiratory failure), and 1 patient had continued TIAs. All of the surviving patients recovered to their neurological baseline.

CONCLUSIONS Patients with MMD presented at a younger age than those in whom aneurysms were more prevalent. Microneurosurgery with either intracranial bypass or aneurysm clipping is extremely challenging but feasible at expert centers in patients with MOPD II, and good long-term outcomes are possible.

http://thejns.org/doi/abs/10.3171/2016.6.PEDS16243

KEY WORDS bypass; intracranial aneurysm; Majewski osteodysplastic primordial dwarfism; moyamoya disease; vascular disorders

I NDIVIDUALS with Majewski osteodysplastic primordial dwarfism Type II (MOPD II) (Online Mendelian Inheritance in Man \#210720) are among the smallest human beings at maturity. Their pleasant outgoing personality, high-pitched nasal voice, and distinctive facial features make them quite recognizable. Their life expectancy is decreased to 20 s or 30 s. In a natural-history study from Hall et al. ${ }^{3}$ of 58 patients with MOPD II or other forms of primordial dwarfism, 4 of 11 deaths were a result of rupture of intracranial vessels.

MOPD II is a rare autosomal recessive genetic disorder caused by mutations in the pericentrin gene or PCNT,

ABBREVIATIONS ACOA = anterior communicating artery; ICA = internal carotid artery; $\mathrm{MCA}=$ middle cerebral artery; $\mathrm{MMD}=$ moyamoya disease; $\mathrm{MOPD}$ II = Majewski osteodysplastic primordial dwarfism Type II; PICA = posterior inferior cerebellar artery; SAH = subarachnoid hemorrhage; STA = superficial temporal artery; TIA = transient ischemic attack; VB = vertebrobasilar.

SUBMITTED May 2, 2016. ACCEPTED June 28, 2016.

INCLUDE WHEN CITING Published online September 9, 2016; DOI: 10.3171/2016.6.PEDS16243. 
which organizes the mitotic spindle. The loss of PCNT impedes cell division, decreases cellularity of the embryo, and leads to severe growth restriction. ${ }^{9}$ Features include proportionate intrauterine growth retardation, poor postnatal growth, extremely small stature (adult height $<100$ $\mathrm{cm}$ ), severe microcephaly, skeletal dysplasia, characteristic facial features, and normal or near-normal intelligence.?

Previous studies ${ }^{1,3}$ have shown that $25 \%-50 \%$ of patients with MOPD II have intracranial vascular anomalies, which include moyamoya disease (MMD) and multiple intracranial aneurysms, both of which can be a major cause of stroke or early death. ${ }^{3}$ Early diagnosis is critical for reducing morbidity and postponing death in these patients, and a screening program using MR angiography has been implemented. ${ }^{1}$ However, because of the patients' extremely small size, diminutive arteries, and narrow anatomical corridors, surgical management of these intracranial anomalies is quite challenging, and few successful surgical cases have been reported in the literature. Here, we present our institutional results for this patient group.

\section{Methods}

A prospectively collected institutional surgical database of children with MOPD II and intracranial vascular anomalies in whom surgery was performed at Stanford University Medical Center between 2005 and 2012 by the senior author (G.K.S.) was analyzed retrospectively. Patients' clinical notes, imaging findings, and operative video recordings were reviewed. Long-term outcome data were obtained from the Potentials Foundation Primordial
Registry, an approved prospective registry of patients with MOPD II located at Nemours/Alfred I. duPont Hospital for Children.

\section{Results}

Ten patients with MOPD II underwent surgery between 2005 and 2012; 5 patients had MMD, 2 had intracranial aneurysms, and 3 had both MMD and aneurysms. The patients presented with transient ischemic attack (TIA) $(\mathrm{n}=$ $2)$, ischemic stroke $(n=2)$, intraparenchymal hemorrhage from MMD $(n=1)$, and aneurysmal subarachnoid hemorrhage $(\mathrm{SAH})(\mathrm{n}=1)$, and 4 were diagnosed on screening (Table 1).

The mean age of the 8 patients with MMD, all of whom underwent extracranial-intracranial revascularization (14 indirect, 1 direct), was 9 years (range $1-17$ years). The diameter of the distal superficial temporal artery (STA) was $0.3-1.0 \mathrm{~mm}$ (mean $0.6 \mathrm{~mm}$ ). There were no postoperative complications after bypass surgery.

The mean age of the 5 patients with aneurysms was 15.5 years (range 9-18 years). Two patients experienced postoperative complications, 1 with transient left-sided weakness after aneurysm clipping (no infarct seen on postoperative diffusion-weighted MRI of the brain) and 1 with femoral thrombosis (after a diagnostic angiogram), which required subsequent surgical repair. Two patients also underwent endovascular coiling of intracranial aneurysms.

During a mean follow-up of 5.9 years (range 3-10 years), 3 patients died (1 as a result of SAH caused by a

TABLE 1. Summary of neurosurgical management and follow-up data in patients with MOPD II and intracranial vascular anomalies

\begin{tabular}{|c|c|c|c|c|c|c|c|c|}
\hline $\begin{array}{l}\text { Case } \\
\text { No. }\end{array}$ & Presentation & $\begin{array}{l}\text { Age } \\
\text { at Op } \\
\text { (yrs) }\end{array}$ & Intracranial Anomaly & $\begin{array}{l}\text { Year } \\
\text { of Op }\end{array}$ & Type of Surgery & $\begin{array}{l}\text { Latest } \\
\text { FU }\end{array}$ & $\begin{array}{l}\text { Yrs of } \\
\text { FU }\end{array}$ & Clinical/Imaging Outcome \\
\hline 1 & Ischemic stroke & $<1$ & MMD & 2009 & Bilateral indirect bypass & 2015 & 6 & Asymptomatic, bypasses patent \\
\hline 2 & Screening & 3 & MMD & 2008 & Bilateral indirect bypass & 2015 & 7 & $\begin{array}{l}\text { Occasional mild TIAs, by- } \\
\text { passes patent }\end{array}$ \\
\hline 3 & Ischemic stroke & 4 & MMD & 2012 & Bilateral indirect bypass & 2015 & 3 & Asymptomatic, bypasses patent \\
\hline 4 & Screening & 9 & MMD & 2009 & Right indirect bypass & 2015 & 6 & Asymptomatic, bypass patent \\
\hline 5 & TIAs & 14 & MMD & 2008 & Bilateral indirect bypass & 2014 & 6 & $\begin{array}{l}\text { Asymptomatic, bypasses pat- } \\
\text { ent; died of MI at } 6 \text { yrs }\end{array}$ \\
\hline \multirow[t]{2}{*}{6} & $\begin{array}{l}\text { Intracranial } \\
\text { hemorrhage }\end{array}$ & 10 & MMD & 2008 & Bilateral indirect bypass & 2012 & 4 & $\begin{array}{l}\text { Asymptomatic, bypasses patent } \\
\text { at } 3 \mathrm{yrs} \text {; died of respiratory } \\
\text { failure at } 4 \text { yrs }\end{array}$ \\
\hline & & & $\mathrm{P}_{1} / \mathrm{P}_{2}$ aneurysm & & Surveillance & & & \\
\hline \multirow[t]{2}{*}{7} & TIAs & 14 & MMD & 2005 & Bilateral indirect bypass & 2015 & 10 & Asymptomatic, bypasses patent \\
\hline & $\mathrm{SAH}$ & 18 & PCA aneurysm & 2009 & Coiling & & & Stable aneurysm neck remnant \\
\hline \multirow[t]{2}{*}{8} & $\mathrm{SAH}$ & 17 & MMD & 2008 & $\begin{array}{l}\text { Right direct bypass, left } \\
\text { indirect bypass }\end{array}$ & 2015 & 7 & Asymptomatic, bypasses patent \\
\hline & & & PICA aneurysm, PCA aneurysm & 2008 & Clipping & & & Stable small PCA aneurysm \\
\hline 9 & Screening & 9 & $\begin{array}{l}\text { ACoA aneurysm (new aneu- } \\
\text { rysms found on FU angio- } \\
\text { gram) }\end{array}$ & 2010 & Clipping & 2015 & 5 & $\begin{array}{l}\text { SAH in 2012; died of SAH } \\
\quad \text { from new aneurysm in } 2015\end{array}$ \\
\hline 10 & Screening & 18 & $\begin{array}{l}4 \text { aneurysms (pericallosal, } M_{1} \\
\text { ACoA, VB) }\end{array}$ & $\begin{array}{l}2010 \\
2012\end{array}$ & Clipping, coiling & 2015 & 5 & Asymptomatic \\
\hline
\end{tabular}

$\mathrm{FU}=$ follow-up; $\mathrm{MI}=$ myocardial infarct; $\mathrm{PCA}=$ posterior cerebral artery. 

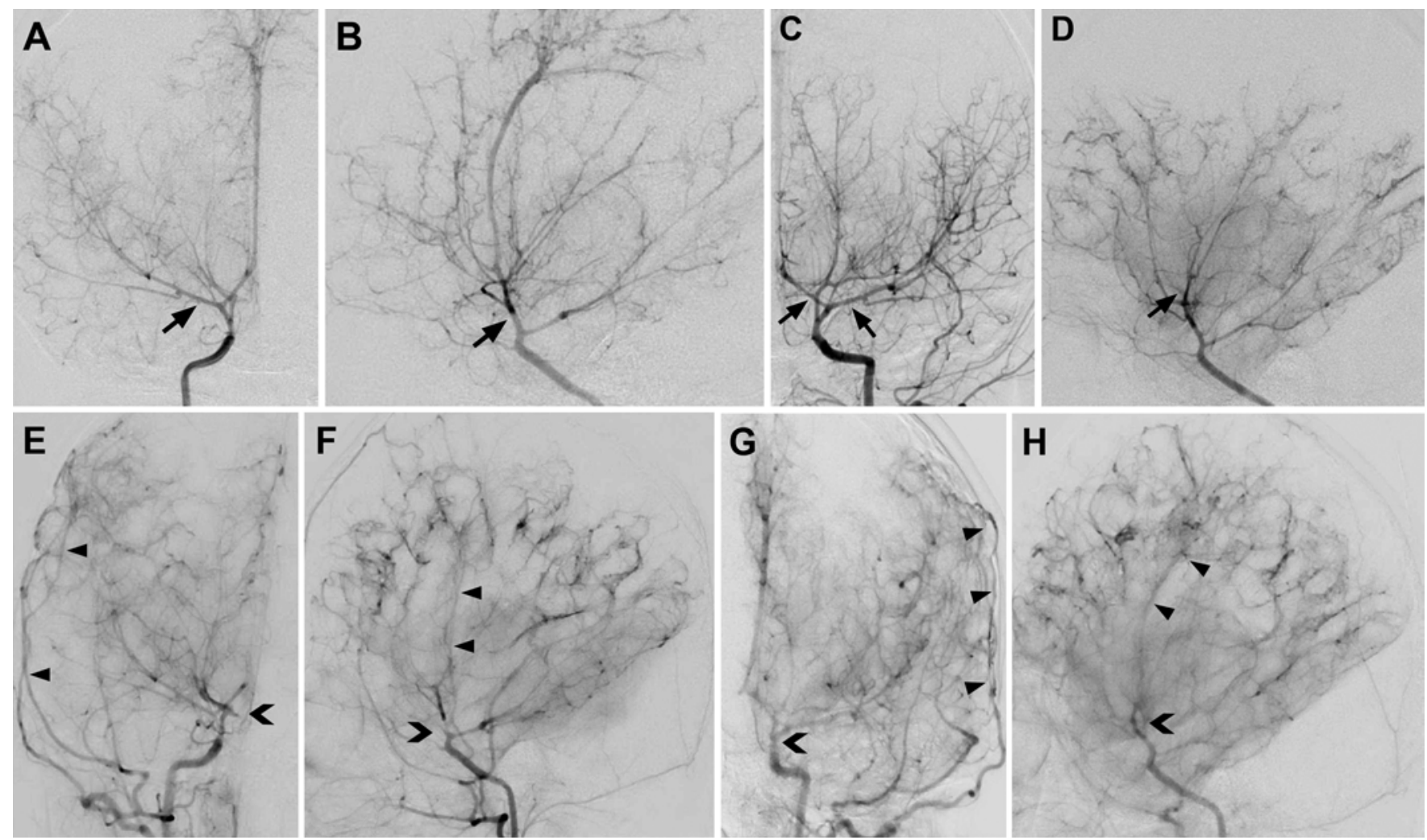

FIG. 1. Case 1. Cerebral angiograms of a 10-month-old girl with MOPD II, craniosynostosis, and MMD, before and after bilateral encephaloduroarteriosynangiosis. Anteroposterior (A) and lateral (B) views of right ICA injection and anteroposterior $(\mathbf{C})$ and lateral (D) views of left ICA injection show bilateral high-grade stenosis of MCAs and anterior cerebral arteries with a network of collateral blood vessels consistent with bilateral MMD (arrows). Anteroposterior $(\mathbf{E})$ and lateral $(\mathbf{F})$ views of right common carotid artery (CCA) injection and anteroposterior $(\mathbf{G})$ and lateral $(\mathbf{H})$ views of left CCA injection 18 months after encephaloduroarteriosynangiosis bypass showing patent bilateral indirect bypass grafts supplying the MCA territory (arrowheads), with progressive stenosis of the bilateral ICAs and moyamoya vessels (chevron arrows).

new untreated aneurysm, 1 as a result of myocardial infarction 6 years after surgery, and 1 as a result of respiratory failure 4 years after surgery), and 1 patient had occasional mild TIAs. All surviving patients recovered to their neurological baseline.

\section{Illustrative Cases}

\section{Case 1}

A 10-month-old girl (initial birth weight $1.0 \mathrm{~kg}$, birth length $35 \mathrm{~cm}$ ) with MOPD II and craniosynostosis presented with a new-onset seizure. Brain MRI showed a left posterior parietal infarct. An angiogram revealed bilateral high-grade stenoses of the middle cerebral arteries (MCAs) and anterior cerebral arteries, with a network of collateral blood vessels consistent with bilateral MMD (Fig. 1A-D). A SPECT scan revealed decreased perfusion in the bilateral parietal occipital regions with no augmentation after acetazolamide challenge, indicating impaired hemodynamic reserve. She underwent uneventful bilateral indirect encephaloduroarteriosynangiosis bypass procedures staged 1 week apart and was discharged after each surgery on Postoperative Day 3. Postoperative angiography at 18 months revealed patent bilateral bypass grafts supplying the bilateral MCA territories with progressive stenosis of the bilateral internal carotid arteries (ICAs)
(Fig. 1E-H). At 2.5 years of age, she also underwent bilateral frontoorbital advancement for craniosynostosis correction. Six years after surgery, she remains neurologically normal.

\section{Case 8}

A 17-year-old boy with MOPD II (weight $13.2 \mathrm{~kg}$, height $100 \mathrm{~cm}$ ) presented with sudden-onset severe headache, nausea, and vomiting consistent with SAH. He then was diagnosed with a ruptured left posterior inferior cerebellar artery (PICA) aneurysm (Fig. 2A), bilateral MMD with supraclinoid ICA stenosis, and extensive collateral vessels (Fig. 3A-D). Brain MRI showed no evidence of infarct, and SPECT revealed a perfusion defect in the right parietal cortex. Over a 3-week period he underwent clipping of the left PICA aneurysm, a right direct extracranial-to-intracranial bypass (using a $0.9-\mathrm{mm}$ STA branch to a $0.9-\mathrm{mm} \mathrm{M}_{4}$ branch of the MCA [Fig. 4]; indocyanine green videoangiography confirmed graft patency), and an indirect left extracranial-to-intracranial bypass. He had an excellent postoperative recovery; angiography 6 months after surgery confirmed complete obliteration of the PICA aneurysm (Fig. 2B) and patent bilateral bypass grafts supplying the majority of the cortical hemispheres (Fig. 3E and F). After 7 years of follow-up, he remained neuro- 

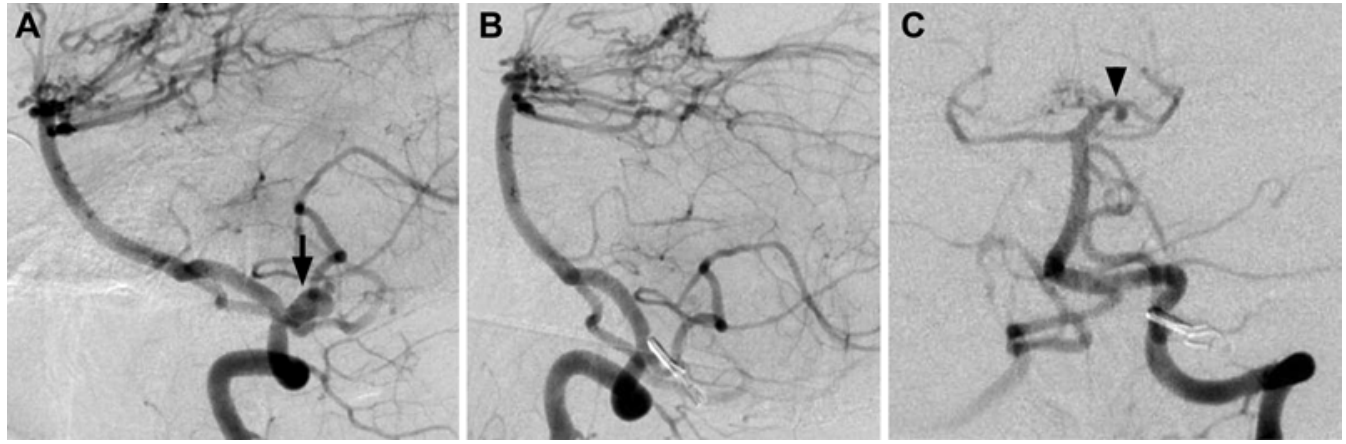

FIG. 2. Case 8. Cerebral angiograms of a 17-year-old boy with MOPD II, PICA aneurysm (before and after clipping), and MMD. A: Lateral view of left vertebral artery injection showing left PICA aneurysm (arrow). B: Complete obliteration of the aneurysm 6 months after surgery. C: Follow-up angiogram (anteroposterior view of vertebral injection) 7 years after surgery showing a 3-mm left $P_{1}$ aneurysm (arrowhead) that remained stable in size in the follow-up period.

logically normal; angiography revealed an asymptomatic 3-mm left $\mathrm{P}_{1}$ aneurysm that had not changed over the 7 years (Fig. 2C).

\section{Case 10}

An 18-year-old boy with MOPD II (weight $12.3 \mathrm{~kg}$, height $83.8 \mathrm{~cm}$ ) and multiple health problems since birth, including gastroesophageal reflux disease, developmental delay, scoliosis (for which he underwent corrective surgery), recurrent urinary tract infections, and chronic renal insufficiency, was found to have 3 intracranial aneurysms during the screening process. He underwent clippings of a 6-mm MCA aneurysm, a 4-mm anterior communicating artery (ACoA) aneurysm, and a $10-\mathrm{mm}$ partially throm-
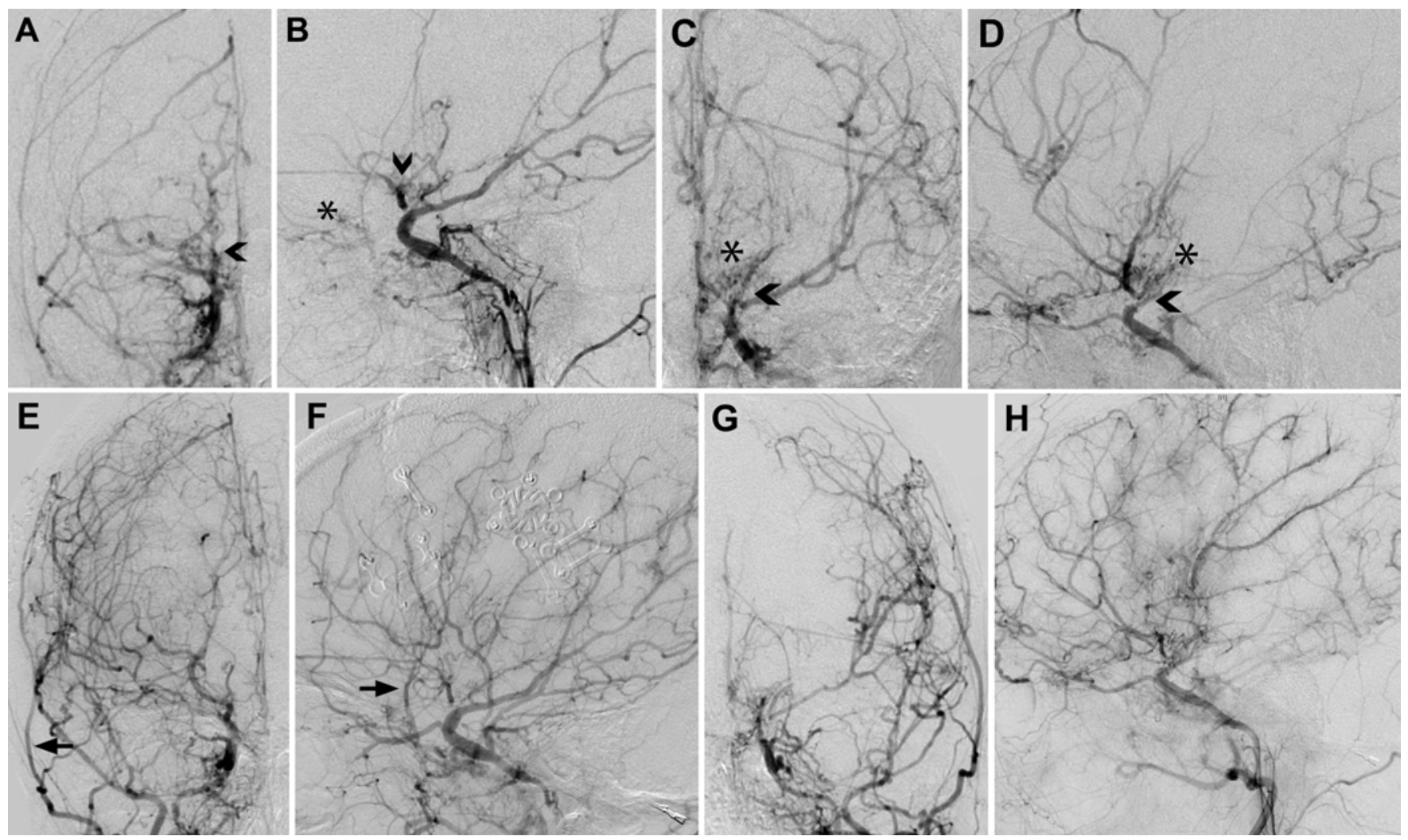

FIG. 3. Case 8. Cerebral angiograms. Preoperative (A-D) and 6-month postoperative (E-F) angiograms showing right direct STA-MCA bypass and left indirect encephaloduroarteriosynangiosis extracranial-to-intracranial bypasses. A and B: Anteroposterior and lateral views, respectively, of right CCA injection. C and D: Anteroposterior and lateral views, respectively, of left CCA injection. Chevron arrows indicate bilateral MMD with supraclinoid ICA stenosis, and asterisks indicate extensive collateral vessels. E and F: Anteroposterior and lateral views, respectively, of right CCA injection showing patent direct STA-MCA bypass graft (arrows). G and H: Anteroposterior and lateral views, respectively, of left CCA injection showing a patent indirect encephaloduroarteriosynangiosis bypass graft. Both bypass grafts supply the majority of the cortical hemispheres. 


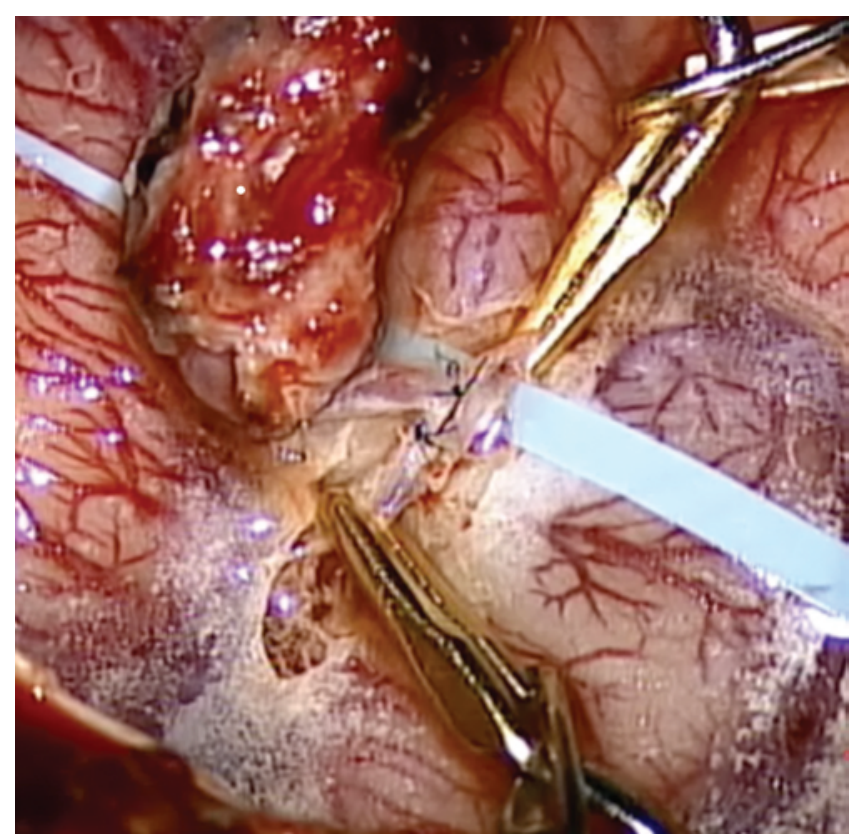

FIG. 4. Case 8. Intraoperative photograph of right direct extracranial-tointracranial bypass (using a 0.9-mm STA branch to $0.9-\mathrm{mm} \mathrm{M}_{4}$ branch of the MCA). Figure is available in color online only.

bosed pericallosal aneurysm (Fig. 5, left). He developed some transient postoperative left-sided weakness, most likely related to retraction injury, which subsequently improved. Magnetic resonance imaging revealed no evidence of postoperative cerebral diffusion-weighted infarcts. Postoperative angiography confirmed complete obliteration of the intracranial aneurysms (Fig. 5 right). Subsequent follow-up angiography 2 years later revealed a new 3-mm vertebrobasilar (VB) aneurysm (Fig. 6B), which was treated with endovascular coil embolization; complete occlusion was achieved (Fig. 6C). After 5 years of follow-up, he was well and had no neurological problems.

\section{Discussion}

Majewski osteodysplastic primordial dwarfism Type II (Online Mendelian Inheritance in Man \#210720) is a distinctive diagnostic entity within the microcephalic primordial dwarfism group and one of the most common conditions encountered. ${ }^{8,13}$ Aside from the classic features of severe prenatal and postnatal growth failure, individuals with MOPD II have a characteristic skeletal dysplasia,,, 12 abnormal dentition, ${ }^{6}$ insulin resistance ${ }^{4}$ and an increased risk for cerebrovascular disease $\mathrm{e}^{1,2,10}$ The disorder is caused by mutations in the PCNT gene and is inherited in an autosomal recessive manner. Moyamoya disease and multiple intracranial aneurysms represent independent sources of morbidity and death in up to 50\% of patients with MOPD II. Some of our patients also exhibited coexistence of the 2 intracranial vascular pathologies.

In our small series, MMD affected the younger patients, whereas older patients developed intracranial aneurysms (which ruptured in 2 patients). Because of the progressive nature of new intracranial vascular anomalies in

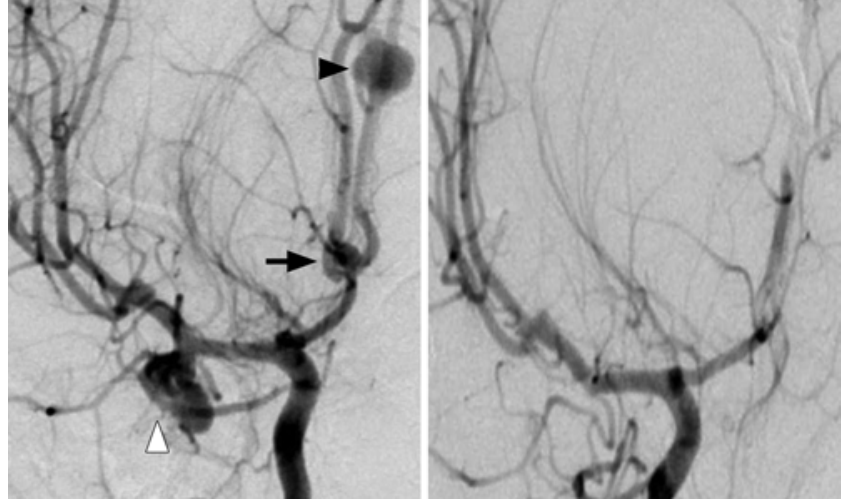

FIG. 5. Case 10. Cerebral angiograms (anteroposterior views of right ICA injection) of an 18-year-old boy with MOPD II. Left: Three intracranial aneurysms, including a 6-mm MCA aneurysm (white arrowhead), 4-mm ACoA aneurysm (arrow), and 10-mm, partially thrombosed left pericallosal aneurysm (black arrowhead). Right: Postoperative angiogram confirming complete obliteration of the 3 intracranial aneurysms.

patients with MOPD II, we recommend long-term vascular imaging surveillance for the development of new vascular anomalies or progression of existing ones. We advocate MR angiography (preferred to reduce the long-term radiation risk) or $\mathrm{CT}$ angiography at diagnosis and then annually for life; cerebral angiograms should be obtained when clinically indicated to study the cerebral vasculature in greater detail.

As found in our case series, 1 patient developed a new aneurysm 2 years after initial imaging. We believed that it was important to treat this aneurysm early even though it was small (less than $7 \mathrm{~mm}$ in diameter). According to International Study of Unruptured Intracranial Aneurysms (ISUIA) data, ${ }^{11}$ aneurysms in the posterior circulation less than $7 \mathrm{~mm}$ in diameter have only a $2.5 \% 5$-year cumulative rupture rate; however, this finding would not necessarily apply to our cohort of patients with MOPD II, because such patients were not included in the ISUIA study. Therefore, we elected to treat the aneurysm aggressively and achieved successful complete occlusion. We developed this proactive aneurysm-treatment strategy after we followed a patient with MOPD II (Case 7) with a posterior cerebral artery aneurysm that ruptured 4 years later and another patient with MOPD II (Case 9) who suffered an SAH from a newly developed basilar artery aneurysm 2 years after clipping of an ACoA aneurysm, with no other aneurysms identified at the time of initial diagnosis. Additional aneurysms were also identified over the 5 years of subsequent follow-up, and she underwent coiling of the ruptured basilar tip aneurysm, and pipeline-assisted coiling of a VB junction aneurysm and left ICA aneurysm. Five other tiny aneurysms were monitored (2 right MCA aneurysms, 1 left MCA aneurysm, 1 right terminal ICA aneurysm, and 1 right posterior communicating artery aneurysm), but unfortunately, this patient died as a result of a ruptured untreated right-sided aneurysm 5 years after her initial diagnosis.

Because the STA and $\mathrm{M}_{4}$ arteries in patients with MOPD II are extremely small and fragile, it is not usually possible to perform a direct bypass, although we did so successfully in 1 patient. The indirect encephaloduroar- 


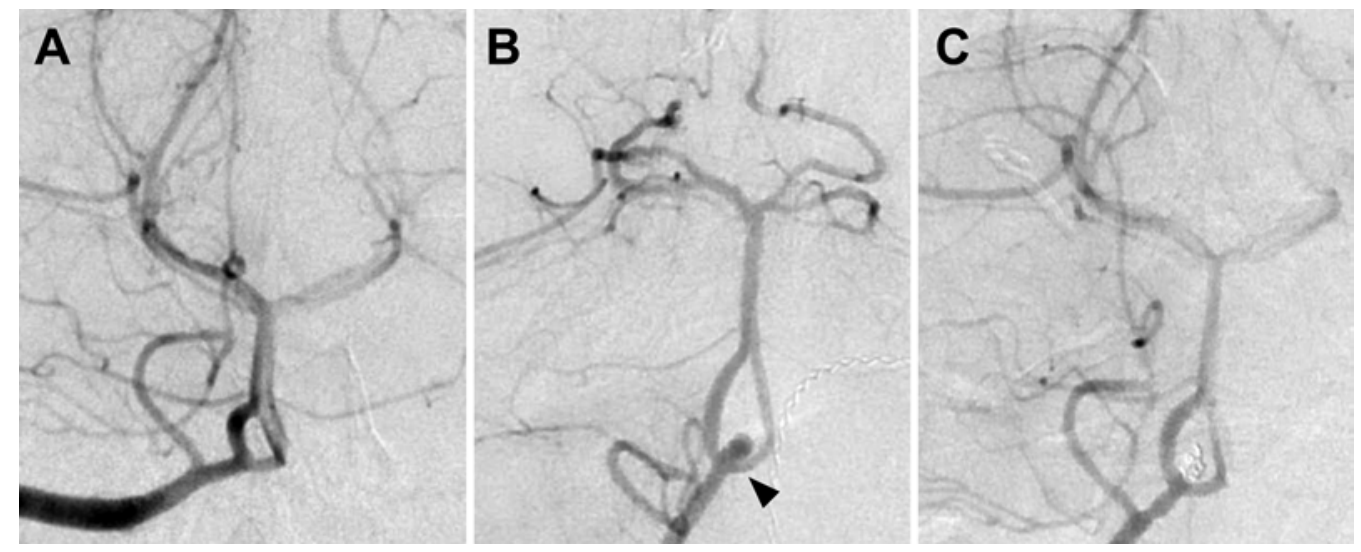

FIG. 6. Case 10. Cerebral angiograms (anteroposterior views of right vertebral artery injection). A: Initial angiogram showing no VB junction aneurysm. B: Follow-up angiogram 2 years later revealing a new 3-mm VB junction aneurysm (arrowhead); note the presence of a fenestrated proximal basilar artery trunk. C: After coil embolization, note complete occlusion of the aneurysm.

teriosynangiosis procedures provided robust angiographic revascularization, according to follow-up angiograms, and excellent short- and long-term neurological outcomes. Few successful neurovascular surgical cases of patients with MOPD II have been reported in the literature. ${ }^{5,10,13}$ Young et al. ${ }^{13}$ and Kannu et al. ${ }^{5}$ separately described 1 case each of a child with MOPD II and MMD. Developmental delay was noted in the child studied by Young et al. ${ }^{13}$ at 2.5 years, and despite surgical attempts to restore the carotid circulation, the child experienced progressive disability and loss of skills caused by ongoing multiple cerebral infarctions. In the case described by Kannu et al., 5 the child developed sudden right-sided weakness at the age of 2 years, 11 months. Cranial imaging confirmed cerebral infarction secondary to MMD and progressive arterial stenosis; she underwent bilateral encephaloduroarteriosynangiosis and had no additional strokes after surgery. However, her fine and gross motor skills were mildly delayed after the previous cerebral ischemic episodes. Waldron et al. ${ }^{10}$ reported 3 cases of patients with MOPD II, multiple intracranial aneurysms, and MMD. Two of the patients (a 15-year-old boy and a 17-year-old girl) presented with aneurysmal SAH, and aneurysm obliteration was performed by either clipping or coiling. One patient had recovered to baseline status by discharge, and 1 died 5 months later of basal ganglia hemorrhage. The third patient was a 22-month-old boy with bilateral MMD who experienced additional strokes despite encephaloduroarteriosynangiosis procedures 6 months earlier. Magnetic resonance imaging revealed new infarcts, and digital subtraction angiography showed bilateral ICA occlusion and patent STA grafts but no revascularization from the onlay grafts. An attempted direct STA- $\mathrm{M}_{2}$ segment bypass failed to establish adequate cerebral blood flow, and temporalis muscle synangiosis and multiple bur hole placements were eventually performed to encourage hemispheric collateralization. No long-term outcome was reported in these cases.

As shown in our case series, these patients often have multiple other comorbidities. With a mean follow-up of 5.9 years (range 3-10 years), 2 of our 10 patients died of other medical problems. Of those remaining, 1 patient with MMD experienced nondisabling symptoms consistent with TIAs, and another patient who had undergone previous aneurysm clipping experienced an aneurysmal SAH from a newly developed aneurysm. Otherwise, at the time of this writing, the rest of the cohort had an active lifestyle and no new neurological symptoms.

Apart from the technical challenges of surgically managing the intracranial vascular anomalies in this group of patients, anesthesia risks are also an important consideration. Many of these children have severe respiratory problems, such as recurrent upper respiratory tract infection and pneumonia, during the newborn period, and scoliosis can compromise their respiratory capacity. ${ }^{3}$ Furthermore, there is increasing evidence that the vascular changes noted intracranially might be part of a more systemic problem, with patients developing coronary artery stenosis, pulmonary artery stenosis, and renal artery aneurysms. ${ }^{1}$ Therefore, the involvement of an expert multidisciplinary team is paramount for managing these patients successfully.

\section{Conclusions}

To our knowledge, this is the largest reported surgical series of patients with MOPD II and intracranial vascular anomalies. Moyamoya disease presented at a younger age, whereas aneurysms were more prevalent in the older age group. Microneurosurgery with either intracranial revascularization or aneurysm clipping is extremely challenging but feasible at expert centers, and good long-term outcomes are possible. Long-term surveillance with vascular imaging is also extremely important in view of the progressive nature of these intracranial vascular anomalies.

\section{Acknowledgments}

We thank Elizabeth E. Hoyte for figure preparation and Cindy H. Samos for manuscript editing.

This work was funded in part by Bernard Lacroute, Ronni Lacroute, the William Randolph Hearst Foundation, and Russell and Beth Siegelman (to Dr. Steinberg). The Primordial Registry at the Nemours/Alfred I. duPont Hospital for Children is funded partially by the Potentials Foundation and the Walking With Giants Foundation. 


\section{References}

1. Bober MB, Khan N, Kaplan J, Lewis K, Feinstein JA, Scott CI Jr, et al: Majewski osteodysplastic primordial dwarfism type II (MOPD II): expanding the vascular phenotype. Am J Med Genet A 152A:960-965, 2010

2. Brancati F, Castori M, Mingarelli R, Dallapiccola B: Majewski osteodysplastic primordial dwarfism type II (MOPD II) complicated by stroke: clinical report and review of cerebral vascular anomalies. Am J Med Genet A 139:212-215, 2005

3. Hall JG, Flora C, Scott CI Jr, Pauli RM, Tanaka KI: Majewski osteodysplastic primordial dwarfism type II (MOPD II): natural history and clinical findings. Am J Med Genet A 130A:55-72, 2004

4. Huang-Doran I, Bicknell LS, Finucane FM, Rocha N, Porter $\mathrm{KM}$, Tung YC, et al: Genetic defects in human pericentrin are associated with severe insulin resistance and diabetes. Diabetes 60:925-935, 2011

5. Kannu P, Kelly P, Aftimos S: Microcephalic osteodysplastic primordial dwarfism type II: a child with café au lait lesions, cutis marmorata, and moyamoya disease. Am J Med Genet A 128A:98-100, 2004

6. Kantaputra P, Tanpaiboon P, Porntaveetus T, Ohazama A, Sharpe P, Rauch A, et al: The smallest teeth in the world are caused by mutations in the PCNT gene. Am J Med Genet A 155A:1398-1403, 2011

7. Majewski F, Goecke TO: Microcephalic osteodysplastic primordial dwarfism type II: report of three cases and review. Am J Med Genet 80:25-31, 1998

8. Rauch A: The shortest of the short: pericentrin mutations and beyond. Best Pract Res Clin Endocrinol Metab 25:125130, 2011

9. Rauch A, Thiel CT, Schindler D, Wick U, Crow YJ, Ekici $\mathrm{AB}$, et al: Mutations in the pericentrin (PCNT) gene cause primordial dwarfism. Science 319:816-819, 2008

10. Waldron JS, Hetts SW, Armstrong-Wells J, Dowd CF, Fullerton HJ, Gupta N, et al: Multiple intracranial aneurysms and moyamoya disease associated with microcephalic osteodysplastic primordial dwarfism type II: surgical considerations.

J Neurosurg Pediatr 4:439-444, 2009

11. Wiebers DO, Whisnant JP, Huston J III, Meissner I, Brown RD Jr, Piepgras DG, et al: Unruptured intracranial aneurysms: natural history, clinical outcome, and risks of surgical and endovascular treatment. Lancet 362:103-110, 2003

12. Willems M, Geneviève $\mathrm{D}$, Borck $\mathrm{G}$, Baumann $\mathrm{C}$, Baujat $\mathrm{G}$, Bieth E, et al: Molecular analysis of pericentrin gene (PCNT) in a series of $24 \mathrm{Seckel} /$ microcephalic osteodysplastic primordial dwarfism type II (MOPD II) families. J Med Genet 47:797-802, 2010

13. Young ID, Barrow M, Hall CM: Microcephalic osteodysplastic primordial short stature type II with café-au-lait spots and moyamoya disease: another patient. Am J Med Genet A 127A:218-220, 2004

\section{Disclosures}

Dr. Steinberg is a consultant for Qool Therapeutics, Peter Lazic US, Inc., and NeuroSave.

\section{Author Contributions}

Conception and design: Steinberg, Teo, Johnson, Marks. Acquisition of data: all authors. Analysis and interpretation of data: Steinberg, Teo, Johnson, Do, Dodd. Drafting the article: Teo, Johnson. Critically revising the article: Steinberg, Teo. Reviewed submitted version of manuscript: Steinberg, Teo, Marks, Do, Dodd, Bober. Approved the final version of the manuscript on behalf of all authors: Steinberg. Administrative/technical/material support: Bell-Stephens.

\section{Correspondence}

Gary K. Steinberg, Department of Neurosurgery, R281, Stanford University Medical Center, 300 Pasteur Dr., Stanford, CA 943055327. email: gsteinberg@stanford.edu. 